\title{
Gibt es ein Menschenrecht auf ethische Bildung?
}

\author{
Is There a Human Right to Ethical Education?
}

\section{GERHARD KRUIP*}

Today, individuals are challenged by a world of growing complexity. They need a lot of capabilities, among them also ethical capabilities like moral sensibility, powers of moral judgement and moral argumentation, moral motivation and moral responsibility. On the other hand, also modern societies need ethically qualified individuals for their own functioning and their social integration. This is even true for the economic subsystem of society. Therefore, the right to education, declared in the UNUniversal Declaration of Human Rights of 1948, also implies a right to ethical education. The article ends giving some indications to the practical consequences for training and continuing education.

Keywords: Human Right, Right to Education, Ethical Education, Economic Ethics

\section{Derwachsende Bedarfethischer Bildung in der,Multioptionsgesellschaft}

Dass die Welt, in der wir leben, komplexer und unübersichtlicher geworden ist, ist ein Allgemeinplatz, dessen Aussage trotzdem richtig ist. Auf Grund der miteinander zusammen hängenden und sich gegenseitig verstärkenden Prozesse der funktionalen Differenzierung, der Globalisierung, der Individualisierung und der Pluralisierung wurden die Einzelnen herausgelöst aus relativ stabilen, überkommenen Lebenszusammenhängen. Die meisten Menschen haben heute viel mehr Alternativen, können und müssen sich immer wieder entscheiden, was sie tun, lassen und denken wollen oder sollen. Selbstverständlich hängt die Zahl der Möglichkeiten auch ab von Herkunft, Geschlecht, Bildung und sozioökonomischen Ressourcen, aber sogar die Ärmsten der Armen besitzen heute mehr Alternativen und Entscheidungsmöglichkeiten wie -zwänge als ihre Vorfahren. Neben vielen anderen zeitdiagnostischen Begriffen (vgl. Schimank et al. 2007), die zur Charakterisierung der Gegenwart verwandt wurden, ist der der ,Multioptionsgesellschaft ${ }^{6}$ besonders gut geeignet, diesen Sachverhalt zu kennzeichnen (vgl. Gross 1994), da er nicht nur die steigende Komplexität beschreibt, sondern zugleich auf die darin enthaltene Dynamik aufmerksam macht:

„Die endlose und kompetitive Ausfaltung neuer Möglichkeiten ist omnipräsent, nicht nur in den Regalen der Supermärkte, sondern auch im Reich des Geistes. Die rasche Folge von die Lebenswirklichkeit als Parallelwelten begleitenden, einander kommentierenden und konkurrierenden Theorie- und Sinnwelten offenbart einen Welt-, Menschen- und Selbstverbesserungszwang, der endlose Folge und weitertreibende Ursache der gleichen gesellschaftlichen Dynamik ist.“ (ebd.: 11)

Prof. Dr. Gerhard Kruip, Kath.-Theol. Fakultät im Fachbereich 01, Lehrstuhl für Christliche Anthropologie und Sozialethik, Saarstraße 21, D-55099 Mainz, Tel.: +49-(0)6131-39-22699, Fax: +49-(0)6131-3926945, E-Mail: kruip@uni-mainz.de, Forschungsschwerpunkte: Bildungsgerechtigkeit, Ethische Bildung, Wirtschaftsethik, Grundfragen der Sozialethik, Lateinamerikanische Theologie und Sozialethik. 
Wer sich in dieser Multioptionsgesellschaft bewegt, steht ständig vor neuen Entscheidungen. Es sind Entscheidungen zu treffen über Fragen, die früher gar nicht zu entscheiden waren, weil einem natürliche Prozesse, gesellschaftliche Konventionen oder autoritative Entscheidungen gar keinen Raum zu eigener Entscheidung ließen. Es stehen Entscheidungen an, für die es bislang keine Modelle gibt, sondern ad hoc neue Lösungen oder mindestens Verfahren zur Annäherung an eine Lösung entwickelt werden müssen. Schlimmer noch: es muss zudem ständig neu entschieden werden, nach welchen Kriterien man sich dabei orientieren möchte, denn es gibt auch eine Vielzahl von Orientierungsangeboten, die alle umstritten bleiben. Es kann nicht immer nur nach dem Kriterium entschieden werden, welche aktuelle Entscheidung zukünftige Entscheidungen möglichst lange offen hält. In früheren Zeiten gaben ethische Werte und moralische Regeln relativ klar vor, nach welchen Kriterien praktische Rationalität vorzugehen habe. Heute ist dies alles offensichtlich sehr viel schwieriger geworden, was u. a. einen Boom an Ethik-Literatur ausgelöst hat. Allerdings ist Gross (1994) hinsichtlich der Chancen zur Orientierung durch Ethik angesichts der ständig noch gesteigerten Vielzahl von Optionen sehr pessimistisch:

\begin{abstract}
„Der Diskurs über die universalistische Ethik (die wie eine Art Krönung euroamerikanischen Fortschrittsprogrammes erscheint), eskortiert das Steigerungsprogramm der Moderne auf einer Seins- und Reflexionshöhe, die die bange Frage laut werden lässt, ob man begriffen haben muß, was ein gutes Leben ausmache, um ein gutes Leben zu leben, (...). Dem Schweigen der Natur und der Beredtheit der Wissenschaft über sie korrespondiert das Schweigen des Menschen und die Beredtheit der ethischen Diskurse. Wie immer die Diskussionen über Moral, Tugend, eine zeitgemäße Ethik ausfallen, sie demonstrieren auf ihre Weise und mit ihren Mitteln jene endlose Optionierung, die nicht nur alle Lebensbereiche und Lebenssphären erreicht, sondern alle Seinsebenen erklommen und erobert hat. Die lautstark angepriesenen rettenden Einsichten und Rezepte sabotieren, indem sie optional verwandt werden, geradewegs sich selber.“ (ebd.: 394-395)
\end{abstract}

Jedoch ist zu fragen, ob diese Skepsis wirklich in diesem Maße angebracht ist. Beobachtet man nämlich Menschen, die sich in ihrer privaten Lebenswelt, in der Öffentlichkeit, der Zivilgesellschaft oder im Beruf ernsthaft über die vielen Optionen Gedanken machen und sich überlegen, was sie tun wollen oder sollen, so drängt sich keinesfalls der Eindruck auf, sie verwendeten ethische Überlegungen immer nur, optional'. Sicher scheinen unübersichtlich viele Veröffentlichungen an ethischer Ratgeberliteratur und philosophischen Arbeiten zum Thema, Lebenskunst' sowie eine gewisse Neuentdeckung ethischer Tugenden zunächst ein Indikator für diese Überbietung der Multioptionsgesellschaft durch Multioptionalität in den ethischen Diskursen zu sein. Lässt man sich jedoch wirklich auf eine Beschäftigung damit ein, so kann man nicht leugnen, dass diese Texte von großer Ernsthaftigkeit geprägt sind. ${ }^{1}$ Und die hohe, weit über philosophische Binnendiskurse hinausgehende Nachfrage nach ihnen lässt sich eigentlich auch nicht anders erklären, als dass die Leser/innen in ihnen auch tatsächlich ethische Orientierung suchen. Dem entsprechen Erfahrungen in Schule, Ausbildung und Fortbildung, wo lebens- und berufsethische Themen zunehmend an Aufmerksamkeit gewinnen. Auch 
konstatieren aktuelle Wertestudien keinesfalls einen Werteverfall oder eine alles erlaubende Beliebigkeit, sondern, insbesondere auch bei Jugendlichen, eine bemerkenswerte Konstanz der für besonders wichtig gehaltenen Werte und moralischen Überzeugungen. ${ }^{2}$ Sicherlich werden moralische Regeln nie wirklich universell akzeptiert sein, sicherlich wird in vielen Entscheidungsfragen eine gewisse Unsicherheit bleiben, was sich wirklich ethisch rechtfertigen lässt, sicherlich wird es immer wieder Menschen geben, die sich in ihrem moralischen Handeln durch überkomplexe Situationen und unübersichtliche Orientierungsangebote entmutigen lassen, aber trotzdem bietet Ethik nach wie vor eine Hilfe beim Versuch, sich dem moralisch Richtigen wenigstens anzunähern und zu jedenfalls vorläufig richtigen Entscheidungen zu kommen.

All dies spricht für einen tatsächlichen Bedarf an Ethik, der mit den erwähnten Herausforderungen einer komplexer werdenden Welt zu tun hat, aber eben - zumindest teilweise - auch so befriedigt werden kann, dass auch unübersichtlichere Gesellschaften, in denen über ethische Fragen auch noch ständig gestritten wird, ein einigermaßen friedliches und hinsichtlich ihrer moralischen Qualität wohl auch nicht erheblich schlechteres Zusammenleben als früher organisieren können, auch wenn dies heute anspruchsvoller geworden ist. Zur eigenen Lebensbewältigung können Menschen heute kaum mehr Konventionen oder Traditionen einfach übernehmen. Dass sie aber in Auseinandersetzung mit ihnen und der Gegenwart eine eigene Lebensorientierung erst entwickeln müssen, ist zugleich eine große Chance für die Ethik. Sie suchen und brauchen Möglichkeiten, ihre moralischen Kompetenzen zu entwickeln und weiter zu entwickeln.

Aber nicht nur die Individuen sind für die erfolgreiche Bewältigung ihres eigenen Lebens auf die Ausbildung ethischer Kompetenzen angewiesen, auch die Gesellschaft braucht Individuen, die sich, bei aller funktionalen Ausdifferenzierung in verschiedene Subsysteme mit ihren Eigenlogiken und diesen Logiken entsprechenden Rollenmustern, trotzdem moralische Kompetenzen bewahren. Dies ist nicht zuletzt an der Weltfinanzkrise 2008-2009 und der durch sie ausgelösten Diskussion über individuelle Verantwortung deutlich geworden. Die Krise zeigte, dass es offenbar nicht möglich ist, durch Rahmenordnungen und Spielregeln alleine sicherzustellen, dass es nicht zu Fehlentwicklungen kommen kann. Erst durch individuelle Sensibilität für ethisch problematische Folgen, nur wenn die Handelnden auch Tugenden ausbilden, werden Regeln eingehalten und wird der Geist dieser Regeln befolgt, auch dann, wenn durch Kontrolle und Sanktionen allein die Einhaltung eben nicht garantiert werden kann. Und wer wollte heute noch eine Gesellschaft, in der Kontrolle und Sanktionen allein wirklich garantieren, dass es nicht zu Fehlentwicklungen kommen kann? Beide Kirchen, immer noch die gesellschaftlich bedeutsamsten moralischen Autoritäten, haben in entsprechenden Erklärungen sowohl auf die Notwendigkeit geeigneter Rahmenbedingungen wie auf die große Bedeutung individueller Verantwortung hingewiesen. ${ }^{3}$ Bei aller not-

$2 \quad$ Vgl. Joas et al. (2005); Klages (2001); Kruip (2001).

3 So in seiner neuen Enzyklika Benedikt XVI. (2009) und die EKD in Rat der Evangelischen Kirche in Deutschland (2009) und schon kurz vorher allgemein mit Blick auf die Verantwortung von Unternehmern der Rat der Evangelischen Kirche in Deutschland (2008). 
wendigen Kritik ${ }^{4}$ zeigt die Rezeption solcher Texte, dass es in dieser Frage nach individueller Verantwortung durchaus eine breite gesellschaftliche Zustimmung gibt.

Das ist auch nicht wirklich verwunderlich, wenn man sich klar macht, wie Menschen unter heutigen gesellschaftlichen Bedingungen Moral lernen. Hierzu sind besonders die Studien von Nunner-Winkler (2001) aufschlussreich. Sie konnte zeigen, dass bereits kleine Kinder ein Wissen um moralische Normen erwerben und verstehen, was es heißt, etwas kategorisch tun oder nicht tun zu sollen. Schon ab zehn Jahren können sie auch entsprechende moralische Prinzipien formulieren.

\begin{abstract}
„Dieser Wissensaufbau verdankt sich universellen Lernmechanismen. Kinder lesen ihr Wissen am Alltagsleben ab: an der Sprachverwendung und an konkreten Unterweisungen, an ihren Interaktionserfahrungen und an den sozialen Institutionen, in die sie hineinwachsen." (Nunner-Winkler 2001: 321)
\end{abstract}

Dass trotzdem der Eindruck eines Werteverfalls entsteht, hängt nach Nunner-Winkler (2001) damit zusammen, dass wir uns im Übergang zwischen traditionellen und modernen Moralvorstellungen befinden, der auch mit einer Veränderung der Motivationsstruktur verbunden ist: „Zunehmend treten rigide Überich-Internalisierungen und eine vorreflexiv geprägte Konformitätsdisposition zurück zugunsten einer ich-näheren Form moralischer Motivation - einer identitätskonstitutiven Selbstbindung an Moral (...) (ebd.: 322). Damit wird Moral zu einer Sache eines postkonventionellen vernünftigen Selbst, das sich im Laufe des Lebens durch Erfahrung und Reflexion immer weiter selbst bildet. „Wir sind alle unser Leben lang moralisch unterwegs“ (Nöstlinger 1980: 1; vgl. Winkler 2008). Solche Prozesse können dann auf vielfältige Weisen, auf die noch zurückzukommen sein wird, unterstützt und gefördert werden.

„Moralische Urteilsfähigkeit (...) lässt sich fördern, lässt sich schulen - durch offene Diskurse unter allen potentiell Betroffenen, durch Einbindung in Entscheidungen und konkrete Verantwortlichkeiten, durch das Aufzeigen der Bedingungen nicht nur eines verträglichen Zusammenlebens im Nahbereich, sondern auch der Funktionsfähigkeit sozialer Systeme.“ (Nunner-Winkler 2005: 191)

Damit rückt die Unterstützung und Förderung ethischer Lernprozesse auch in den Bereich der Bildung und der für Bildung zuständigen gesellschaftlichen Institutionen. „Moral ist lehrbar“ (Lind 2003). Ist die Notwendigkeit ethischen Lernens im Rahmen von Bildungsprozessen erkannt, so kann gefragt werden, inwieweit aus einem allgemeinen Menschenrecht auf Bildung auch so etwas wie ein Menschenrecht auf ethische Bildung abgeleitet werden kann.

\title{
2. Das Menschenrecht auf Bildung
}

Menschenrechte gelten unabhängig von staatlicher Setzung, sie sind universell gültig, unverletzlich und unveräußerlich. Trotzdem tragen rechtliche Kodifizierung und faktische Akzeptanz einen Zeitindex. Die Formulierung von Menschenrechten reagiert auf historische Unrechtserfahrungen, auf gesellschaftliche Situationen mit bestimmten, aus Gerechtigkeitsgründen zu berücksichtigenden Bedürfnissen und Nöten (vgl. Biele-

$4 \quad$ Zu meiner Kritik an „Caritas in Veritate“ siehe Kruip (2009a). 
feldt 1998). So hängt auch das Menschenrecht auf Bildung sowohl mit anthropologischen Grundbedingungen wie mit einer gesellschaftlichen Situation zusammen, die ein Menschenrecht auf Bildung dringlich macht.

Die Begründung eines solchen Menschenrechts auf Bildung 5 wird deshalb zwar auch anthropologisch ansetzen und zeigen, dass der Mensch ein bildbares, bildungsfähiges und auf Bildung angewiesenes Wesen ist. In einem weiteren Schritt muss aber gezeigt werden, dass dies unter Bedingungen heutiger, moderner, globalisierter, hochkomplexer Gesellschaften in besonderem Maße gilt. So ist Bildung unabdingbare Voraussetzung der Subjektwerdung und gesellschaftlicher Beteiligung sowie in beiderlei Hinsicht notwendige Voraussetzung der Freiheit. Weil alle Menschen Bildung für ein menschenwürdiges Leben brauchen, muss ihnen auch ein Recht auf Bildung zugestanden werden, zunächst allgemein als moralisches Recht, dann aber auch präzisiert und kodifiziert als positives und einklagbares Recht.

Man kann sogar sagen, dass in der heutigen, Wissensgesellschaft ${ }^{6}{ }^{6}$ die Frage der Bildung zur neuen ,Sozialen Frage' wird. Der Begriff der Sozialen Frage war bekanntlich schon im 19. Jahrhundert entstanden, als der Wandel von einer noch feudal und ständisch geprägten Agrargesellschaft zur kapitalistischen Industriegesellschaft einsetzte und viele Menschen ihrer herkömmlichen Lebens- und Ernährungsmöglichkeiten beraubte. Das damit verbundene soziale Elend zwang die westlichen Industriegesellschaften zu einem Lernprozess, dessen Ergebnis darin bestand, dass nach und nach soziale Sicherungssysteme zur Bekämpfung der schlimmsten Armut und zur Abfederung der sozialen Risiken einer kapitalistischen Marktwirtschaft geschaffen wurden. War die Soziale Frage damals die ,Arbeiterfrage', so ist sie heute die ,Frage der Bildungsgerechtigkeit'. In einer Wirtschaft, die in wachsendem Maße von modernen Kommunikationstechnologien geprägt ist, in der immer schneller neueste wissenschaftliche Ergebnisse umgesetzt werden, die sich zunehmend weltweit vernetzt und deren hochkomplexe Arbeitsabläufe ein vielseitigeres Kompetenzprofil erfordern, haben gering Qualifizierte immer weniger Chancen, einen Arbeitsplatz zu finden. Aber auch für andere Lebensbereiche - von der politischen Partizipation über die Beteiligung am kulturellen Leben bis hin zur persönlichen Lebensgestaltung - wird die eigene Ausstattung mit Bildungsgütern immer bedeutsamer. An der Verteilung des Zugangs zu Bildungsangeboten und der effektiven Möglichkeiten zu ihrer Wahrnehmung entscheidet sich zunehmend, ob die Verhältnisse einer Gesellschaft als ,gerecht ${ }^{\circ}$ gelten können. Wenn der Zugang zu Bildung in einer Gesellschaft ungleich verteilt ist, wenn insbesondere diese ungleiche Verteilung von Eigenschaften wie Wohnort, Herkunft, Sprachkenntnissen, finanziellen Ressourcen der Eltern etc. abhängt, dann handelt es sich um eine soziale Ungerechtigkeit.

Um insbesondere diese sozialethischen Fragen aufzuarbeiten, die sich in der aktuellen Bildungsdebatte stellen, haben Marianne Heimbach-Steins (in Bamberg) und ich (am Forschungsinstitut für Philosophie Hannover) in einem von der Deutschen Forschungsgemeinschaft finanzierten Projekt seit Anfang 2006 bis einschließlich Februar

$5 \quad$ Vgl. hierzu insbesondere Kunze (2007) und Kunze (2008).

$6 \quad$ Vgl. Filipovic et al. (2003) und Filipovic (2007). 
2009 Fragen der Bildungsgerechtigkeit und sozialethischer Kriterien zu ihrer Umsetzung in Deutschland unter dem Fokus des ,Menschenrechts auf Bildung' untersucht. ${ }^{7}$ Ein menschenrechtlicher Zugang zum Thema Bildungsgerechtigkeit, wie er auch durch den Deutschland-Besuch des UN-Sonderberichterstatters für das Menschenrecht auf Bildung, Vernor Muñoz, im Februar 2006 und seinen im März 2007 veröffentlichten Bericht ins Bewusstsein der Öffentlichkeit gehoben wurde ${ }^{8}$, hat mehrere Vorteile: In der Allgemeinen Erklärung der Menschenrechte (AEMR) der UNO von $1948^{9}$ und weiteren Kodifizierungen in diversen Konventionen und Abkommen hat man eine eindeutige, teilweise rechtlich verankerte und so gut wie universell gültige Grundlage, auf die man sich berufen kann und von der her die Kritik an mangelnder Bildungsgerechtigkeit mit größerem Nachdruck vertreten werden kann.

Nach Art. 26 der AEMR hat jeder

„das Recht auf Bildung. Die Bildung ist unentgeltlich, zum mindesten der Grundschulunterricht und die grundlegende Bildung. Der Grundschulunterricht ist obligatorisch. Fach- und Berufsschulunterricht müssen allgemein verfügbar gemacht werden, und der Hochschulunterricht muss allen gleichermaßen entsprechend ihren Fähigkeiten offen stehen.“ (Art. 26, Abs. 1)

Zunächst ist das Menschenrecht auf Bildung ein negatives Recht: Niemand darf daran gehindert werden, sich zu bilden und entsprechende Bildungsmöglichkeiten in Anspruch zu nehmen. Aber es ist auch ein positives Recht, und zwar im Bereich grundlegender Bildung. Diese ist obligatorisch, was das Menschrecht auf Bildung der Kinder gegen möglicherweise andere Interessen der Eltern schützt, und muss unentgeltlich zur Verfügung gestellt werden. Aus der Formulierung des Menschenrechts auf Bildung wird aber auch deutlich, dass es nicht nur um ein jedem zustehendes Minimum gehen kann. Zumindest in einer Gesellschaft, die über ausreichende Ressourcen verfügt, darf es auch bei aller Bildung, die darüber hinausgeht, keine anderen Zugangsbeschränkungen geben als die jeweilige Eignung. Die gleiche Forderung ergibt sich auch schon aus dem allgemeinen Diskriminierungsverbot des Art. 2 AEMR:

„Jeder Mensch hat Anspruch auf die in dieser Erklärung verkündeten Rechte und Freiheiten ohne irgendeine Unterscheidung, wie etwa nach Rasse, Farbe, Geschlecht, Sprache, Religion, politischer oder sonstiger Überzeugung, nationaler oder sozialer Herkunft, nach Eigentum, Geburt oder sonstigen Umständen (...).“

Dabei müssen alle nicht nur formale, sondern reale Chancen des Zugangs zu den Bildungseinrichtungen haben, die ihrer Eignung entsprechen. Nach der AEMR gibt es mit Art. 28 sogar ein sehr bemerkenswertes, allgemeines positives Recht auf Instituti-

$7 \quad$ Im Folgenden greife ich auch auf einige Ergebnisse aus unserer Arbeit zurück. Vgl. HeimbachSteins et al. (2003); Heimbach-Steins et al. (2007); Heimbach-Steins et al. (2008); HeimbachSteins et al. (2009a), Heimbach-Steins et al. (2009b). Die beiden wichtigsten Arbeiten, die Dissertation von Katja Neuhoff und die Habilitationsschrift von Axel-Bernd Kunze, werden in diesem oder spätestens im nächsten Jahr erscheinen. Siehe auch www.menschenrecht-auf-bildung.de. Im Folgenden verwende ich auch Textpassagen aus dem bald erscheinenden Aufsatz Kruip (2009b).

8 Siehe insbesondere Overwien et al. (2007); Muñoz (2007).

9 Z. B. zu finden auf www.igfm.de/Allgemeine-Erklaerung-der-Menschenrechte.89.0.html (2.5.09). 
onen und Verhältnisse, in denen die Realisierung dieser allgemeinen Menschenrechte möglich ist:

„Jeder Mensch hat Anspruch auf eine soziale und internationale Ordnung, in welcher die in der vorliegenden Erklärung angeführten Rechte und Freiheiten voll verwirklicht werden können.“

Man kann sich natürlich fragen: Ist Bildung überhaupt etwas, worauf man ein Recht haben kann? Kann man Bildung ,einklagen'? Aus pädagogischer Perspektive wird an einer Forderung eines Rechts auf Bildung häufig Kritik geübt. ${ }^{10}$ Ein solches Recht als einen Anspruch anderen oder dem Staat gegenüber könne es im strengen Wortsinn allein deshalb nicht geben, weil Bildung letztlich immer Selbstbildung sein müsse. Überhaupt stelle der sozialethische Blick auf Fragen der Bildung, des Lernens und des pädagogischen Handelns eine Verkürzung dar. Allenfalls könne es um ein Recht auf Ermöglichung von Bildung gehen, wobei sich die materiellen und personellen Ressourcen, die dafür notwendig seien, einem standardisierten und empirisch überprüfbaren Zugriff entzögen. Richtig daran ist sicherlich, dass sich Bildung im klassischen Verständnis kaum empirisch messen lässt und deshalb auch eine zu weit gehende Verrechtlichungen des Bildungsprozesses problematisch wäre. Es ist aber doch ausgesprochen plausibel, dass für diejenigen, bei denen in empirischen Untersuchungen große Rückstände in Schlüsselqualifikationen wie Lesen, Textverständnis und mathematischem Denken nachgewiesen werden, aller Wahrscheinlichkeit nach auch die Möglichkeiten zu einer Bildung im klassischen Verständnis kaum gegeben sein dürften.

Es ist klar, dass in Deutschland sehr wohl das Menschenrecht auf Bildung in erheblichem Umfang verletzt wird, wenn Kinder, illegal' in Deutschland lebender Migranten aus Angst vor Entdeckung nicht zur Schule gehen bzw. geschickt werden. In manchen Bundesländern gibt es für sie entgegen dem Menschenrecht auf Bildung nicht einmal eine Schulpflicht (vgl. Neuhoff 2008). Aber auch die Tatsache, dass Bildungserfolg und Bildungszugang so stark vom Bildungshintergrund der Eltern abhängen, stellt eine menschenrechtlich nicht legitimierbare Diskriminierung dar (vgl. Kruip/Neuhoff 2008), zumal auch empirisch nachgewiesen ist, dass bei den vielen Übergängen, die in Deutschland von einer Schulform zur nächsten bewältigt werden müssen, keinesfalls immer nur nach Eignung, sondern sehr häufig - bewusst oder unbewusst - nach der Bildungsferne des Elternhauses entschieden wird. Besonders eklatant ist dieses Phänomen bei den Schullaufbahnempfehlungen, die Lehrer/innen Kindern für den Übergang von der Grundschule in eine weiterführende Schule geben. Aber auch die von Eltern oder Kindern selbst gesetzten Bildungsziele orientieren sich weniger an der Leistung als an den sozialen Erwartungen des Elternhauses und seines Umfeldes. Unter Bildungsforschern ist inzwischen weitgehend Konsens, dass sich solche diskriminierenden Faktoren innerhalb einer Bildungskarriere umso stärker bemerkbar machen, je mehr solche Schwellen zu überwinden sind, weil sich die Selektionsfaktoren multiplizieren. ${ }^{11}$

Zur Konkretisierung des Menschenrechts auf Bildung ist es nun natürlich wichtig zu klären, was hier mit Bildung gemeint ist. Art. 26 gibt selbst eine Antwort: vor allem

11 Hierzu insbesondere Ditton (2007). 
„Grundschulunterricht und die grundlegende Bildung". Sicherlich sind darunter der Erwerb elementarer Kulturtechniken wie Lesen, Schreiben und Rechnen, Grundkenntnisse zur Orientierung in gegenwärtigen Gesellschaften, die Beherrschung mindestens einer Sprache und weitere Schlüsselqualifikationen gemeint, die alle eine wesentliche Voraussetzung dafür bieten, eine Berufsausbildung zu absolvieren, einen Arbeitsplatz zu bekommen, sich am demokratischen und kulturellen Leben zu beteiligen usw. Doch umfasst diese Bildung auch ethische Bildung?

\section{Ein Menschenrecht auf ethische Bildung als Implikation des Menschen- rechts auf Bildung}

Das Menschenrecht auf Bildung, wie es in der AEMR formuliert worden ist, ist eindeutig offen für ein Bildungsverständnis, das sich keinesfalls auf eine Bildung im Sinne einer Ausbildung in grundlegenden Kulturtechniken oder für eine spätere Berufstätigkeit begrenzen würde. Denn es heißt dort explizit im Art. 26, Abs. 2:

„Die Bildung muss auf die volle Entfaltung der menschlichen Persönlichkeit und
auf die Stärkung der Achtung vor den Menschenrechten und Grundfreiheiten
gerichtet sein. Sie muss zu Verständnis, Toleranz und Freundschaft zwischen allen
Nationen und allen rassischen oder religiösen Gruppen beitragen und der Tätig-
keit der Vereinten Nationen für die Wahrung des Friedens förderlich sein.“

Es geht hier also durchaus auch um eine Bildung im Sinne des klassischen Bildungsbegriffs. Das kann auch gar nicht anders sein, wenn man sich vor Augen führt, dass das Menschenrecht auf Bildung letztlich aus der Menschenwürde heraus begründet werden muss, diese Menschenwürde aber den Selbstzweck des Menschen und seiner Bildungsvollzüge impliziert.

Eine Konsequenz daraus ist, dass es nicht nur ein Menschenrecht auf Bildung geben muss, sondern im Sinne der Achtung vor der Würde aller an Bildung Beteiligter auch ein Menschenrecht in der Bildung und auch Menschenrechte durch Bildung. ,Menschenrechte in der Bildung' sind notwendig, damit auch die Rechte der Kinder, der Jugendlichen, der Eltern und der Lehrer/innen im Bildungsprozess selbst geachtet werden, weil sonst Bildung im Sinne der Bewusstseinsbildung für Menschenrechte selbstwidersprüchlich würde. Mit ,Menschenrechte durch Bildung ist gemeint, dass Bildung erheblich dazu beitragen kann und soll, dass Menschen ihre Rechte kennen, das Bewusstsein entwickeln, sie gegebenenfalls auch in Anspruch nehmen zu wollen und zugleich um Verfahren wissen, die dazu dienen können, entsprechende Rechte auch einzuklagen.

Eine Menschenrechtsbildung, die zur „Stärkung der Achtung vor den Menschenrechten und Grundfreiheiten " führt ist selbst schon ethische Bildung, haben doch die Menschenrechte einen eminent moralischen Charakter. Darüber hinaus ist keine „volle Entfaltung der menschlichen Persönlichkeit“ denkbar, ohne dass sich diese Persönlichkeit auch als ein moralisches Subjekt begreift, das über moralische Kompetenzen verfügen muss. 
Dies wird auch im „Internationalen Pakt über wirtschaftliche, soziale und kulturelle Rechte“ (1966 verabschiedet, 1976 in Kraft getreten) ${ }^{12}$, in dessen Artikel 13 ausführliche Bestimmungen zum Menschenrecht auf Bildung enthalten sind, im Wesentlichen bekräftigt, auch wenn hier nicht explizit von ,ethischer Bildung' die Rede ist. Insbesondere wird hier eigens betont,

„daß die Bildung auf die volle Entfaltung der menschlichen Persönlichkeit und des Bewußtseins ihrer Würde gerichtet sein und die Achtung vor den Menschenrechten und Grundfreiheiten stärken muß. Sie [die Staaten] stimmen ferner überein, daß die Bildung es jedermann ermöglichen muß, eine nützliche Rolle in einer freien Gesellschaft zu spielen, daß sie Verständnis, Toleranz und Freundschaft unter allen Völkern und allen rassischen, ethnischen und religiösen Gruppen fördern sowie die Tätigkeit der Vereinten Nationen zur Erhaltung des Friedens unterstützen muß.“ (Art. 13, Abs. 1)

In Textnr. 5 der ,General Comments ${ }^{6}$ dazu ${ }^{13}$ werden als wichtige Erziehungsziele auch die Gleichheit der Geschlechter und die Bewahrung der Umwelt genannt. Dabei besteht kein Zweifel, dass die Staaten auch mit dafür zuständig sind, dafür zu sorgen, dass Bildungsprozesse auf diese ethischen Inhalte und Ziele hin ausgerichtet werden.

Also ist klar: Die Bildung, die im Menschenrecht auf Bildung gemeint ist, umfasst auch ethische Bildung. Damit impliziert das Menschenrecht auf Bildung auch ein Menschenrecht auf ethische Bildung. ${ }^{14}$

\section{Perspektiven der Umsetzung eines Menschenrechts auf ethische Bildung}

Allgemein gilt für das Menschenrecht auf Bildung, was auch sonst für die Garantie von Menschenrechten im Mittelpunkt steht: es ist zunächst die Rolle des Staates zu bedenken, auch wenn selbstverständlich durch Menschenrechte auch nicht-staatliche Akteure verpflichtet werden. Für die Präzisierung der staatlichen Verantwortung hat sich in der Arbeit der UN-Kommissionen, in den entsprechenden Kommentierungen und in der Literatur der Dreiklang ,respect - protect - fulfill ${ }^{6}$ herausgebildet. ${ }^{15} \mathrm{Zu}$ nächst hat der Staat selbst die Menschenrechte zu respektieren (respect). Diese ,Achtungspflichten' verbieten ihm, durch irgendwelche Vorschriften oder Maßnahmen Menschen daran zu hindern, Bildungsmöglichkeiten, auf die sie ein Recht haben, in

Der Text des einschlägigen Art. 13 findet sich online u. a. auf http://de.wikisource.org/wiki/ Internationaler_Pakt_\%C3\%BCber_Wirtschaftliche,_Soziale_und_Kulturelle_Rechte\#Artikel_13.

13 ,General Comments` des UN-Ausschusses CESCR E/C.12/1999/10 vom 8.12.1999, Textnr. 4. Online unter www.fao.org/righttofood/kc/downloads/vl/docs/AH353.pdf. Vgl. Deutsches Institut für Menschenrechte (2005).

Das Verhältnis von Menschenrechten und Pflichten wird in diesem Beitrag nicht eigens thematisiert. Aber es dürfte klar sein, dass der Anspruch auf Persönlichkeitsentwicklung und ethische Bildung nur realisiert werden kann, wenn das sich bildende Subjekt auch selbst (mehr oder weniger explizit) eine moralische Pflicht zur Selbstbildung und dabei auch der ethischen Bildung für sich erkennt.

Offenbar war es Asbjorn Eide, der als erster in einem ,Report on the Right to Food as a Human Right ${ }^{`}$ diese drei Pflichtenarten von Staaten herausgearbeitet hat. Siehe UN-Dokument E/CN.4/Sub.2/1987/23 (7. Juli 1987), para. 66-69. 
Anspruch zu nehmen. Hier greift auch das schon erwähnte allgemeine Diskriminierungsverbot. Eine entsprechende Aussage, die hier sogar explizit von ethischer Bildung spricht, findet sich auch in Art 13 (3) des ,Internationalen Paktes über wirtschaftliche, soziale und kulturelle Rechte'. Hier verpflichten sich die Staaten, „die Freiheit der Eltern (...) zu achten, (...) die religiöse und sittliche Erziehung ihrer Kinder in Übereinstimmung mit ihren eigenen Überzeugungen sicherzustellen“. Der Staat hat darüber hinaus aber auch ,Schutzpflichten', d. h. die Aufgabe, Menschen davor zu schützen (protect), dass andere deren Rechte verletzen. Er muss also einschreiten, wenn bestimmte gesellschaftliche Gruppen oder Akteure, z. B. auch die Eltern von Kindern, den Zugang zu Bildungsmöglichkeiten in unzulässiger Weise einschränken. Sollten drittens innerhalb der Gesellschaft auf Grund von privater oder zivilgesellschaftlicher Initiative nicht genügend Bildungsmöglichkeiten zur Verfügung gestellt werden, so muss dies der Staat selbst tun. Er hat ,Erfüllungspflichten“'(fulfill), um ausreichend Bildungsmöglichkeiten bereitzustellen. Er hat dabei eine Gewährleistungsverantwortung, was aber nicht bedeutet, dass er immer durch eigene Einrichtungen oder Organisationen Bildung anbieten wird, er muss allerdings dafür sorgen, dass dies überhaupt getan wird.

Bei der weiteren Suche nach Kriterien der Umsetzung positiver Rechte hat sich ein ,4-A-Schema' als besonders fruchtbar erwiesen, das in UN-Menschenrechtsausschüssen entwickelt und durch die Bildungsberichterstatter der UNO auf das Menschenrecht auf Bildung angewandt worden ist. Es umfasst Verfügbarkeit (availability), Zugänglichkeit (accessibility), Annehmbarkeit (acceptability) und Adaptierbarkeit (adaptability). ${ }^{16}$ Verfügbarkeit meint, dass überhaupt Bildungseinrichtungen in ausreichender Zahl zur Verfügung stehen. Diese müssen dann allerdings auch für diejenigen, die sie benötigen, zugänglich sein. Das allein würde jedoch noch nicht ausreichen, wenn die Art des Bildungsangebots aus der Perspektive der zu bildenden Menschen aus moralischen bzw. religiösen Gründen oder aus Mangel an Qualität nicht akzeptabel wäre. Schließlich müssen Bildungsangebote so gut wie möglich auf die Bedürfnisse der zu bildenden Personen ausgerichtet sein, insbesondere ihre Sprache, ihren lebensweltlichen Hintergrund, ihre späteren beruflichen Möglichkeiten usw. berücksichtigen. Bildung ist nämlich nur dann möglich, wenn das Angebot zu den Bildungsnachfragern passt.

Was würde es nun bedeuten, diese Überlegungen zur Umsetzung des Menschenrechts auf Bildung auf ein Menschenrecht auf ethische Bildung zu beziehen? Der Respekt vor diesem Recht zwingt dem Staat eine gewisse Zurückhaltung gegenüber Erziehungsinstanzen wie v. a. der Familie auf. Denn diese sind auf Grund der größeren Nähe zum Alltag sowie zur Lebenswelt, auf Grund des persönlicheren Charakters von Beziehungen und wegen der zu respektierenden Vielfalt konkreter ethischer Lebensentwürfe eher in der Lage, einen Beitrag zur ethischen Bildung zu leisten. Die Schutzpflichten stehen jedoch in einer gewissen Spannung hierzu, denn sie verlangen vom Staat, dafür zu sorgen, dass nicht unmoralische, mit den Freiheitsansprüchen anderer inkompatible Lebensentwürfe durch Erziehung propagiert werden, v. a. dann, wenn sich die betroffenen Kinder oder Jugendlichen nicht dagegen wehren können. So muss der Staat 
einschreiten, wenn bestimmte religiös oder ideologisch motivierte Gruppen von ,Fundamentalisten' eine Erziehung zum Hass, zum Rassismus, zur Verachtung menschlicher Würde etc. propagieren. Kinder und Jugendliche müssen vor einer ,Bildung ' geschützt werden, die zu Menschenrechtsverletzungen aufrufen würde. Um nur ein Beispiel zu nennen: Es ist sicherlich überaus problematisch, Kinder und Jugendliche in die Obhut von Bildungseinrichtungen zu geben, die von der rechtskatholischfundamentalistischen Piusbruderschaft getragen werden. Es ist klar, dass es nicht einfach ist, dies in jedem Einzelfall zu konkretisieren. Hier gibt es legitimer Weise Grauzonen und kontroverse Bereiche.

Schwierig ist auch eine Konkretisierung der Erfüllungspflichten des Staates. Staatliche Bildungseinrichtungen müssen heute zwar viele Erziehungsdefizite primärer Sozialisationsinstanzen ausgleichen, sind dafür aber nicht besonders gut ausgerüstet. Nicht umsonst haben sich die Staaten während der UN-Dekade für Menschenrechtsbildung (1995-2004) mit der Entwicklung und Umsetzung von Konzepten schwer getan und das Meiste zivilgesellschaftlichen Organisationen überlassen (vgl. Mihr 2005). Mit dem 2005 beschlossenen ,UN-Weltprogramm für Menschenrechtsbildung werden jedoch auch der Staat und seine Bildungseinrichtungen explizit zu stärkeren Anstrengungen verpflichtet:

„In den kommenden Jahren sollen die staatlichen Behörden die ,Hauptdarsteller' sein, wenn es darum geht, Menschenrechtsbildung in allen Schul- und Ausbildungsformen umzusetzen - unabhängig davon, ob es sich um Grund-, Berufs- oder weiterführende Schulen handelt.“ (Mihr 2008: 36)

Eine fundierte Menschenrechtsbildung besteht nun allerdings nicht in einer bloßen Information über Menschenrechtserklärungen und internationale Menschenrechtspakte. Es muss auch über Werthintergründe, Begründungsfragen, über konfligierende Rechte und moralische Motivation gesprochen werden. Menschenrechtsbildung ist auch ethische Bildung. ,Hauptdarsteller ${ }^{6}$ zu sein impliziert jedoch wohl nicht unbedingt, dass alle entsprechenden Aktivitäten vom Staat ausgehen müssen und ihre Durchführung staatlicherseits genau vorgegeben sein muss. Um die Pluralität von Weltanschauungen zu respektieren, wird der Staat manches, was über ein Minimum an moralischen Regeln, die auch im Wesentlichen im Rechtssystem einer Gesellschaft verankert sind, hinausgeht, eher zivilgesellschaftlichen bzw. religiösen Trägern oder den Individuen in freier Selbstgestaltung überlassen. Er ist allerdings befugt und aufgerufen, dafür geeignete Freiraume zu schaffen, z. B. durch Religions- und Ethikunterricht an Schulen, durch die Integration berufsethischer Fragestellungen in Ausbildungsgänge oder durch ethische Reflexionsangebote in einem Studium generale, zu denen verschiedenste Akteure eingeladen und zu Beiträgen aufgefordert werden.

Aus der Einsicht heraus, dass ethische Bildung heute das ganze Leben begleiten und deshalb auch in Konzeptionen ,Lebenslangen Lernens' seinen Platz finden muss, insofern dieses nicht allein Aus-, Fort- und Weiterbildung, sondern auch Persönlichkeitsbildung umfasst, haben wir an einem Projekt zum Thema ,Ethisches Lernen in der allgemeinen Erwachsenenbildung ${ }^{6}$ mitgewirkt. ${ }^{17}$ Ziel des Projektes ist es, ethische und von der Katholischen Bundesarbeitsgemeinschaft für Erwachsenenbildung getragen. Die Er- 
Bildung nicht als zusätzliches Angebot, sondern als Querschnittsaufgabe der allgemeinen Erwachsenenbildung fest zu verankern. Da in ganz ähnlicher Weise ethische Fragen als Querschnittsaufgaben in beruflichen Ausbildungsgängen zu realisieren wären, scheint es mir angebracht, auf einige zentrale Ansätze und Erfahrungen aus diesem Projekt auch hier kurz einzugehen.

Ethisch relevante Fragen treten nämlich in alltäglichen kulturellen Zusammenhängen, in Sozialisations- und Kommunikationsprozessen und insbesondere in allen möglichen Bildungszusammenhängen auf, zumeist allerdings in Form impliziter Themen und Lerngelegenheiten, die jedoch explizit gemacht und zu ethischem Lernen genutzt werden können. Fragen des Sollens und Dürfens, des Erlaubten und Verbotenen sind in den unterschiedlichsten Kursen und Seminaren Gegenstand des Gesprächs, werden jedoch häufig nur beiläufig behandelt, manchmal sogar bewusst ausgeblendet oder verdrängt, jedenfalls nicht ausreichend als explizite Gelegenheiten für ethisches Lernen genutzt. Teilnehmern/innen entgeht damit eine wichtige Möglichkeit ihr Lebensund Daseinswissen nicht nur beiläufig, sondern durch ausdrückliche, methodisch kontrollierte Denk- und Lernprozesse zu erweitern. ${ }^{18}$

Wir haben dem Projekt einen offenen Begriff, ethischen Lernens' zugrunde gelegt, der psychische Entwicklungsprozesse und emotionale Komponenten nicht ausblendet, aber einen gewissen Akzent legt auf vernunftmoralische Argumentationskompetenz. Ethisches Lernen umfasst danach gleichermaßen den Zuwachs an ethisch relevantem Wissen wie die Weiterentwicklung ethischer Kompetenz. Es lässt sich in vier Dimensionen beschreiben: Erstens muss ethisch relevantes Wissen erworben werden, damit ein moralisches Problem in einer gegebenen Situation überhaupt erkannt werden kann. Dann braucht es zweitens die Sensibilität, um sich durch ein moralisches Problem selbst herausgefordert zu sehen, drittens die Fähigkeit, sich mit Hilfe argumentativer Prozesse ein moralisches Urteil zu bilden und viertens die Motivation, auch diesem moralischen Urteil gemäß zu handeln. Ethisches Lernen hat dann stattgefunden, wenn ein moralisches Problem aufgrund erweiterten Wissens oder gestiegener Kompetenz in einem der genannten Bereiche nach dem Lernprozess besser bearbeitet werden kann als zuvor.

Zur Förderung ethischen Lernens wurde ein Konzept der Moderation und Förderung ethischer Lernprozesse entwickelt, das einen vierstufigen Prozess vorsieht, der die Komponenten Entdecken, Initiieren, Begleiten und Ergebnissicherung umfasst. Ausgangspunkt bildet ein konkreter moralischer Konflikt, der im Kursgeschehen entweder implizit auftritt oder als Teil des Kursthemas ausgewählt wurde. Nachdem ein

gebnisse werden Anfang des nächsten Jahres in Form eines Sammelbandes, hgg. von Helga Gisbertz, Gerhard Kruip und Markus Tolksdorf im W. Bertelsmann-Verlag, voraussichtlich unter dem Titel ,Ethisches Lernen in der allgemeinen Erwachsenenbildung' veröffentlicht werden. Ich verwende hier Teile aus Kruip/Winkler (2008). Zu allgemeinen grundsätzlichen Informationen zum Projekt siehe unser ,Basispapier‘ Kruip/Winkler (2007), Winkler (2008) sowie die Angaben auf www.ethisches-lernen.de.

18 Das Projekt, Ethisches Lernen in der allgemeinen Erwachsenenbildung hat dazu geeignete Modelle entwickelt, Kursleitende entsprechend geschult und anschließend in einer breiten Erprobungsphase die Reichweite solcher Modelle für die Entwicklung von Urteilskompetenz, Überzeugungen, Einstellungen und Werthaltungen erforscht. 
impliziter Ausgangspunkt entdeckt worden ist, muss er zum Thema gemacht und damit ein Prozess initiiert werden, durch den der moralische Konflikt einer ethischen Reflexion zugänglich gemacht werden kann. Die Dozenten/innen haben dann die Aufgabe, den angestoßenen ethischen Diskurs durch notwendige Differenzierungen, Erklärungen, Ergänzungen und theoretische Bezüge zur Ethik zu begleiten. Den Abschluss des Prozesses bildet die Ergebnissicherung, die z. B. daraus bestehen kann, erreichte Konsense bzw. bestehen bleibende Dissense festzuhalten. Mit diesem sowohl situativen wie progressiven Ansatz wird besonders der Motivationsstruktur erwachsener Lernender, die sich durch die zentrale Bedeutung des persönlichen Zugangs zum Bildungsinhalt auszeichnet, oder der Entwicklungsstufe des moralischen Bewusstseins Erwachsener, die in der Regel bereits über ausgeprägte Überzeugungsund Gewohnheitsmuster verfügen, Rechnung getragen.

Solche Überlegungen können sehr wohl auch in Ausbildungsgänge, beispielsweise in das Hochschulstudium, übertragen werden, und zwar auch in Veranstaltungen, die gerade nicht ein ethisches Thema explizit behandeln, sondern gerade in solche, in denen ethische Themen mitschwingen, aber auch offensiv angesprochen werden können. Gerade für die Wirtschafts- und Unternehmensethik gilt ja, dass es sich um Fragestellungen handelt, die sich aus konkreten Problemkonstellationen ergeben, am besten konkret in diesem Kontext bearbeitet werden und wohl dadurch am ehesten die Chance besteht, dass ethische Einsichten auch umgesetzt werden. Allerdings fehlt es oft an der moralischen Sensibilität zur Wahrnehmung der ethischen Implikationen und an der Fähigkeit, diese Fragen auf einem Niveau zu reflektieren, das den sonst üblichen intellektuellen Standards des Faches entspricht. Würde man für die wirtschafts- und unternehmensethische Ausbildung der Betriebs- und Volkswirtschaftler/innen einzig und allein auf wirtschafts- und unternehmensethische Sonderveranstaltungen setzen, die ohne klare Bezüge zum sonstigen Ausbildungsgang zu absolvieren wären, so entstünde die Gefahr, dass hier ein von den Kernfächern relativ isolierter Lernbereich mit einer ganz anderen Denkkultur etabliert würde, der bei den Studierenden möglicherweise nicht mit der gleichen Ernsthaftigkeit studiert würde, sondern als eine Art Orchideenfach innerhalb der Disziplin nicht wirklich ernst genommen würde. Deshalb erscheint es notwendig, sich auch in den volks- und betriebswirtschaftlichen Kerngebieten und den dafür vorgesehen Lehrveranstaltungen Zeit für die Auseinandersetzung mit impliziten ethischen Fragen zu nehmen, auch hier also die ethische Reflexion als Querschnittsaufgabe zu implementieren. Zugleich dürfte aber eine solche Integration alleine nicht ausreichen, um der Komplexität wirtschafts- und unternehmensethischer Fragen insgesamt gerecht zu werden, den Studierenden eine echte Teilnahme an den internationalen wirtschafts- und unternehmensethischen Diskursen zu ermöglichen und das hohe Niveau der dabei notwendigen, philosophischen Rationalität sichtbar und erlernbar zu machen. Deshalb plädiere ich für eine Kombination beider Ansätze: die Etablierung eines eigenen Pflichtfaches ,Wirtschafts- und Unternehmensethik' im betriebs- und volkswirtschaftlichen Studium sowie die Integration ethischer Fragen als Querschnittsaufgabe in alle Fächer dieses Studiums.

Entscheidend ist dabei aber die Kompetenzentwicklung der Dozenten/innen, welche durch eine Sensibilisierung für implizite ethische Fragestellungen, die Beschäftigung mit der Analyse ethischen Sprechens, mit Modellen der Argumentations- und Kontextanalyse 
sowie mit ethischem Grundlagenwissen über prominente ethische Argumentationsmuster und ethische Theorien gefördert werden müssen, um den hohen Anforderungen eines solchen Konzepts gerecht werden zu können. Auf Seiten der Dozenten/innen ist ein Zuwachs an ethischer Wahrnehmungskompetenz und Argumentationskompetenz notwendig, um ihre Professionalität zu steigern und damit das bildungspolitische Ziel der Qualitätsentwicklung und der dafür ebenfalls notwendigen Reflexion ethischer Implikationen zu unterstützen. Insofern stehen Wirtschafts- und Unternehmensethiker/innen auch vor der Aufgabe, die eigenen Kollegen/innen in Betriebs- und Volkswirtschaft nachhaltig in unternehmens- und wirtschaftsethischen Fragen fortzubilden.

\section{Literaturverzeichnis}

Bayert々, K. (2004): Warum überhaupt moralisch sein?, München: C. H. Beck.

Benedikt XVI. (2009): Caritas in Veritate - Enzyklika von Papst Benedikt XVI. an die Bischöfe, an die Priester und Diakone, an die Personen gottgeweihten Lebens, an die christgläubigen Laien und an alle Menschen guten Willens über die ganzheitliche Entwicklung des Menschen in der Liebe und in der Wahrheit, Bonn: Sekretariat der Deutschen Bischofskonferenz (Verlautbarungen des Apostolischen Stuhls, 186).

Bielefeldt, H. (1998): Philosophie der Menschenrechte - Grundlagen eines weltweiten Freiheitsethos, Darmstadt: WiBuGe.

Deutsches Institut für Menschenrechte (Hrsg.) (2005): Die „General Comments“ zu den VNMenschenrechtsverträgen - Deutsche Übersetzung und Kurzeinführungen, BadenBaden: Nomos Verlagsgesellschaft.

Ditton, H. (Hrsg.) (2007): Kompetenzaufbau und Laufbahnen im Schulsystem - Ergebnisse einer Längsschnittuntersuchung an Grundschulen, Münster et al.: Waxmann.

Filipovic, A. (2007): Öffentliche Kommunikation in der Wissensgesellschaft - Sozialethische Analysen, Bielefeld: W. Bertelsmann.

Filipovic, A./ Kunze, A.-B. (Hrsg.) (2003): Wissensgesellschaft - Herausforderungen für die Christliche Sozialethik, Münster: Lit.

Gross, P. (1994): Die Multioptionsgesellschaft, Frankfurt a. M.: Suhrkamp.

Heimbach-Steins, M./ Kruip, G. (Hrsg.) (2003): Bildung und Beteiligungsgerechtigkeit - Sozialethische Sondierungen, Bielefeld: W. Bertelsmann.

Heimbach-Steins, M./ Kruip, G./ Kunze, A.-B. (Hrsg.) (2007): Das Menschenrecht auf Bildung und seine Umsetzung in Deutschland: Diagnosen - Reflexionen - Perspektiven, Bielefeld: W. Bertelsmann.

Heimbach-Steins, M./ Kruip, G./ Kunze, A.-B. (Hrsg.) (2009a): Bildung, Politik und Menschenrecht - Ein ethischer Diskurs, Bielefeld: Bertelsmann (Forum Bildungsethik, 6).

Heimbach-Steins, M./ Kruip, G./ Kunze, A.-B. (Hrsg.) (2009b): Bildungsgerechtigkeit - Interdisziplinäre Perspektiven, Bielefeld: Bertelsmann.

Heimbach-Steins, M./ Kruip, G./ Neuhoff, K. (Hrsg.) (2008): Bildungswege als Hindernisläufe Zum Menschenrecht auf Bildung in Deutschland, Bielefeld: W. Bertelsmann.

Höffe, O. (2007): Lebenskunst und Moral - Oder macht Tugend glücklich?, München: Beck.

Horster, D. (2004): Was soll ich tun? - Moral im 21. Jahrhundert, Leipzig: Reclam.

Joas, H./ Wiegandt, K. (Hrsg.) (2005): Die kulturellen Werte Europas, Frankfurt a. M.: Fischer.

Klages, H. (2001): Brauchen wir eine Rückkehr zu traditionellen Werten?, in: Aus Politik und Zeitgeschichte, H. B 29, 7-14. 
Kruip, G. (2001): Werteverlust - Wertewandel - Wertepolitik: Ressourcen für eine postkonventionelle Moral, in: Hösle, V. et al. (Hrsg.): Jahrbuch für Philosophie des Forschungsinstituts für Philosophie Hannover, Bd. 12, Wien: Passagen, 137-155.

Kruip, G. (2009a): Entwicklung und Wahrheit - Die Sozialenzyklika Benedikts XVI. ermöglicht viele Lesarten, in: Herder Korrespondenz, Jg. 63, H. 8, 388-392.

Kruip, G. (2009b): Menschenrecht auf Bildung? Diagnosen - Reflexionen - Perspektiven seiner Umsetzung in Deutschland, in: Bock, V. (Hrsg.): Die Würde des Menschen unantastbar? 60 Jahre Allgemeine Erklärung der Menschenrechte, Münster: Lit (im Erscheinen).

Kruip, G./ Neuboff K. (2008): Hauptschüler ohne Zukunftsperspektive?, in: Stimmen der Zeit, Jg. 133, H. 8, 507-529.

Kruip, G./ Winkler, K. (2008): Bildungsethik - Ethische Bildung, in: Natur und Geist - Das Forschungsmagazin der Johannes Gutenberg-Universität Mainz, Jg. 24, H. 1, 40-43.

Kruip, G./ Winkler K. (2007): Ethisches Lernen in der allgemeinen Erwachsenenbildung (Basispapier), Bonn: Katholische Bundesarbeitsgemeinschaft für Erwachsenenbildung.

Kun₹e, A.-B. (2007): Unverzichtbar für die Subjektwerdung des Menschen - Gehalt und Grenzen des Menschenrechts auf Bildung, in: Heimbach-Steins, M./ Kruip, G./ Kunze, A.B. (Hrsg.): Das Menschenrecht auf Bildung und seine Umsetzung in Deutschland: Diagnosen - Reflexionen - Perspektiven, Bielefeld: W. Bertelsmann, 177-197.

Kunze, A.-B. (2008): Das Menschenrecht auf Bildung, in: Münk, H. J. (Hrsg.): Wann ist Bildung gerecht? Ethische und theologische Beiträge im interdisziplinären Kontext, Bielefeld: Bertelsmann, 49-72.

Ladenthin, V. (2009): Ist Bildung notwendig?, in: Heimbach-Steins, M./ Kruip, G./ Kunze, A.B. (Hrsg.): Bildung, Politik und Menschenrecht - Ein ethischer Diskurs, Bielefeld: Bertelsmann (Forum Bildungsethik, 6), 69-80.

Lind, G. (2003): Moral ist lehrbar - Handbuch zu Theorie und Praxis moralischer und demokratischer Bildung, München: Oldenbourg Schulbuchverlag.

Mihr, A. (2005): Die UN-Dekade für Menschenrechtsbildung - Eine Bilanz, in: Der Bürger im Staat, Jg. 55, H. 1, 51-56.

Mihr, A. (2008): Die Vereinten Nationen und Menschenrechtsbildung, in: Aus Politik und Zeitgeschichte, Jg. 30, H. 46, 33-38.

Muñoz, V. (2007): Das Recht auf Bildung in Deutschland, in: Heimbach-Steins, M./ Kruip, G./ Kunze, A.-B. (Hrsg.): Das Menschenrecht auf Bildung und seine Umsetzung in Deutschland: Diagnosen - Reflexionen - Perspektiven, Bielefeld: W. Bertelsmann, 69-96.

Neuboff, K. (2008): Das Menschenrecht auf Bildung für Migrant/inn/en realisieren ..., in: Münk, H. J. (Hrsg.): Wann ist Bildung gerecht? - Ethische und theologische Beiträge im interdisziplinären Kontext, Bielefeld: Bertelsmann, 215-230.

Nöstlinger, Ch. (1980): Moralisch unterwegs, in: Kursbuch, H. 60 (Moral), 1-6.

Nunner-Winkler, G. (2001): Moralische Bildung, in: Wingert, L./ Günther, K. (Hrsg.): Die Öffentlichkeit der Vernunft und die Vernunft der Öffentlichkeit - Festschrift für J. Habermas, Frankfurt a. M.: Suhrkamp, 314-343.

Nunner-Winkler, G. (2005): Zum Verständnis von Moral - Entwicklungen in der Kindheit, in: Horster, D./ Oelkers, J. (Hrsg.): Pädagogik und Ethik, Wiesbaden: Verlag für Sozialwissenschaften, 173-192.

Overwien, B./ Prengel, A. (Hrsg.) (2007): Recht auf Bildung - Zum Besuch des Sonderberichterstatters der Vereinten Nationen in Deutschland, Oplanden, Farmington Hills: Budrich.

Pauer-Studer, H. (2003): Einführung in die Ethik, Wien: WUV (UTB Philosophie, 2350). 
Poenitsch, A. (2009): Bildung - Menschenrecht - Reformpolitik: Überlegungen zu deren Verhältnis, in: Heimbach-Steins, M./ Kruip, G./ Kunze, A.-B. (Hrsg.): Bildung, Politik und Menschenrecht - Ein ethischer Diskurs, Bielefeld: Bertelsmann (Forum Bildungsethik, 6), 17-32.

Rat der Evangelischen Kirche in Deutschland (2008): Unternehmerisches Handeln in evangelischer Perspektive - Eine Denkschrift des Rates der Evangelischen Kirche in Deutschland, Gütersloh: Gütersloher Verlagshaus.

Rat der Evangelischen Kirche in Deutschland (2009): Wie ein Riss in einer hohen Mauer - Wort des Rates der EKD zur globalen Finanzmarkt- und Wirtschaftskrise, Hannover: EKD.

Schimank, U./Volkmann, U. (Hrsg.) (2007): Soziologische Gegenwartsdiagnosen I - Eine Bestandsaufnahme, Wiesbaden: Verlag für Sozialwissenschaften.

Schmid, W. (1998): Philosophie der Lebenskunst - Eine Grundlegung, Frankfurt a. M.: Suhrkamp.

Tomasevski, K. (1999): Preliminary Report of the Special Rapporteur on the Right to Education, Ms. Katarina Tomasevski, submitted in accordance with Commission on Human Rights Resolution 1998/33, Economic and Social Council. E/CN.4/1999/49 (zugänglich über http:/ / ap.ohchr.org/documents/alldocs.aspx?doc_id=1480).

Winkler, K. (2008): „Wir sind alle unser Leben lang moralisch unterwegs“ - Ethisches Lernen in der allgemeinen Erwachsenenbildung, in: Münk, H. J. (Hrsg.): Wann ist Bildung gerecht? - Ethische und theologische Beiträge im interdisziplinären Kontext, Bielefeld: Bertelsmann, 308-331. 